\section{REACH NEW PATIENTS AND WATCH YOUR PRACTICE GROW}

The expert team from Munroe Sutton will be on hand at this year's British Dental Conference and Exhibition advising attendees on the many ways they can help a dental practice grow. Munroe Sutton delivers a new way to attract patients through marketing your dental practice via some of the world's largest financial, healthcare and insurance institutions, local unions and trade associations.

With over 30 years' experience developing and managing dental plans, Munroe Sutton is passionate about ensuring dentists receive fair compensation. At the show,

\section{EXPERT DIGITAL SOLUTIONS}

Whether you are a student, newly qualified or an experienced professional you're all welcome to Stand B04 to meet Henry Schein Dental's friendly and knowledgeable team of experts.

Their specialist teams can offer advice on all aspects of surgery and decontamination equipment, lasers, digital technology, servicing and business support. They will also guide you through the latest developments in surgery design and are happy to support you with the move to a digital workflow, be that chairside or laboratory based. the team will be on hand to share their expertise helping delegates explore the exciting ways that working with Munroe Sutton can help to grow a practice.

If you're searching for an innovative way of reaching new patients, that also ensures payment at the time of service and a team of professionals that is committed to supporting you and your staff, make sure you visit the Munroe Sutton stand at the British Dental Conference and Exhibition this May.

To find out more, call 0808234 3558 or visit www.munroesutton. co.uk

For those looking to integrate digital into practice, Connect Dental from Henry Schein Dental helps dentists access all elements of the digital workflow, from digital impressions to final restoration, including hardware and materials, allowing every area of your practice to be seamlessly linked together.

To get the most out of the latest technology and help future-proof your practice, visit Henry Schein Dental on Stand B04. More information at www.henryschein.co.uk or call 087001020 41. Alternatively you can find the team on Twitter: @HenryScheinUK or Facebook: HenryScheinUK.

\section{WHAT IF YOUR CHILD HAD TOOTHACHE AND NO HOPE OF HELP?}

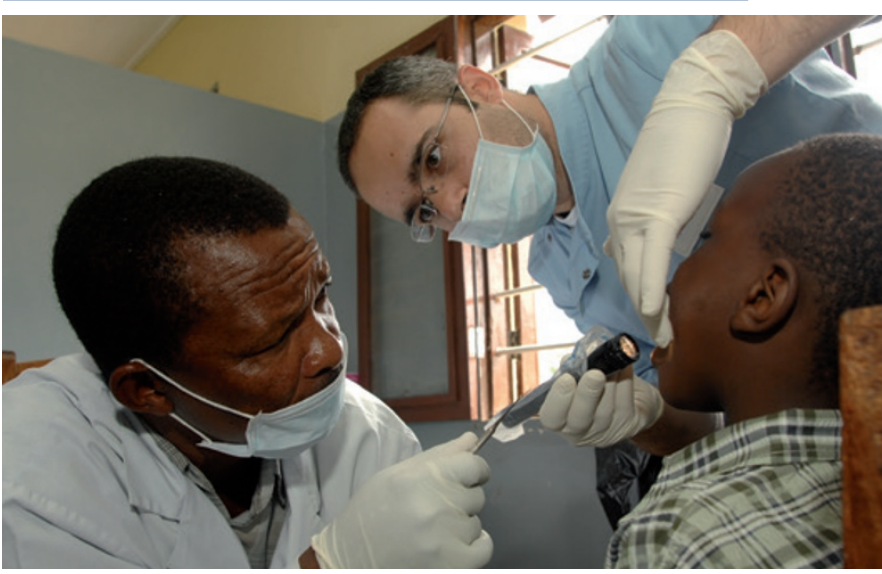

Dental charity Bridge2Aid hopes to increase vital support this year, making it possible to provide access to safe emergency dental treatment for millions more people in need in East Africa.

Chief Executive Mark Topley commented: 'With not even the most basic dental service available for the majority of people living in the rural areas of places like Tanzania, there are countless untreated dental problems. And more than half of people with toothache and no access to basic treatment will develop complications; sometimes, very sadly and shockingly, the complications lead to death.'

However, Bridge2Aid is making a big impact - by training local health professionals already based in villages to extract teeth and relieve pain. And it works - over the past 10 years Bridg2Aid has demonstrated success in both Tanzania and Rwanda, making access to treatment available to over 3 million people. Health professionals trained by Bridge2Aid have shown they can immediately address $98 \%$ of dental problems.

To find out how you can help, visit the team at stand D20 at the British Dental Conference and Exhibition in Manchester, where you can be sure of a warm welcome. Alternatively, visit www.bridge2aid.org/whatif.

\section{HOW MUCH TIME DO YOU TAKE TO LOOK AFTER YOUR OWN HEALTH?}

At the 2015 British Dental Conference and Exhibition, Dentists' Provident will be demonstrating their focus on your health and wellbeing, by providing hands-on sessions to help with your preventive care programmes. Last year alone Dentists' Provident paid over 1,300 claims, with a fifth of those for musculoskeletal issues.

At the Manchester Central Convention Centre on 7-9 May you can find the team from Dentists' Provident on stand B01. They will be accompanied by qualified and experienced practitioners in reflexology and pilates who can discuss your current health concerns.
IAS Academy's pathway was put together by the team behind the Inman Aligner, who have been protocols for years. Your patients will be reassured knowing you have received the highest standard of education, from simple to comprehensive orthodontics. Professor Ross Hobson will be delivering training at the advanced level for ClearSmile Brace.

More information will be available from the IAS Academy team; find them at stand C37, or for further information visit www. iasortho.com, email info@iasortho. com or call 08453665477 . 